\title{
NEW AND MISCLASSIFIED PLANETARY NEBULAE
}

\author{
L. KOHOUTEK \\ Hamburg Observatory, Hamburg-Bergedorf, Germany
}

This fourth supplementary list to the CGPN (Perek, Kohoutek, 1967) contains 81 new objects (Table 1) which were published mainly between 1987 and 1990. We did not include as new PN those objects, which are in a transition phase between $A G B$ and PN (no emission lines), and possible post-PN namely objects having central stars on the evolutionary way to WD and without nebulae. The possible pre-PN are summarized in a separate incomplete list as an Appendix to Table 1.

In Table 2 it is suggested to remove 86 objects from the CGPN or from the previous supplementary lists of PN ( Paper I,II,III). In case of SS (symbiotic stars) we do not remove them from PN because their symbiotic characteristics do not exclude their simultaneous classification as PN. Even the red continuum including TiO bands cannot be a reason for removing such objects from the list of PN. We are convinced that there exist some SS (especially of type D), which can also be classified as PN. It seems that there exist emission line objects which can be called "symbiotic proto-PN". We neither removed some of those objects, for which the following statements were reported: (a) the IRAS spectrum does not correspond to PN, (b) small or compact H II regions, (c) $\mathrm{H} \alpha$ emission line only, (d) no detected objects.

The number of new and misclassified PN differs strongly from that given by Acker and Stenholm (1990) which cen be explained by the different classification criteria used (see Paper II and III). Inspite of the fact that the objects called planetaries very probably do not represent a uniform group, the decision about the question "what is or what is not a PN" would require not only a sufficient observational material, but also the correct understanding of the phenomenon "planetary nebula".

References:

Acker A., Stenholm B., 1990, Astron.Astrophys.Suppl.86, 219

Kohoutek L., 1978, IAU Symp.No.76 (ed.Y.Terzian), p.47. (Paper I)

Kohoutek L., 1983, IAU Symp.No.103 (ed.D.R.Flower),P.17. (Paper II)

Kohoutek L., 1989, IAU Symp.No.131 (ed.S.Torres-Peimbert), p.29. (Paper III)

Perek L., Kohoutek L., 1967, Catalogue of Galactic Planetary Nebulae, Academia Praha. (CGPN) 